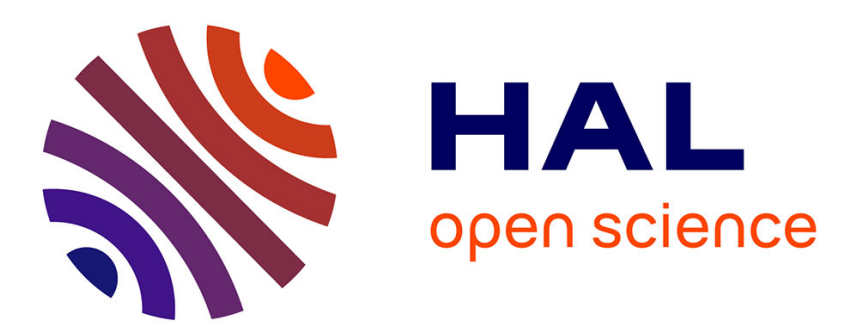

\title{
Chaotic Dynamics of Red Blood Cells in a Sinusoidal Flow
}

Jules Dupire, Manouk Abkarian, Annie Viallat

\section{To cite this version:}

Jules Dupire, Manouk Abkarian, Annie Viallat. Chaotic Dynamics of Red Blood Cells in a Sinusoidal Flow. Physical Review Letters, 2010, 104 (16), pp.168101. 10.1103/PhysRevLett.104.168101 . hal00538828

\section{HAL Id: hal-00538828 \\ https://hal.science/hal-00538828}

Submitted on 24 Nov 2010

HAL is a multi-disciplinary open access archive for the deposit and dissemination of scientific research documents, whether they are published or not. The documents may come from teaching and research institutions in France or abroad, or from public or private research centers.
L'archive ouverte pluridisciplinaire HAL, est destinée au dépôt et à la diffusion de documents scientifiques de niveau recherche, publiés ou non, émanant des établissements d'enseignement et de recherche français ou étrangers, des laboratoires publics ou privés. 


\title{
Chaotic Dynamics of Red Blood Cells in a Sinusoidal Shear Flow
}

\author{
Jules Dupire ${ }^{1,2,3}$, Manouk Abkarian ${ }^{4}$, and Annie Viallat ${ }^{1,2,3 *}$ \\ 1.Adhesion et inflammation lab., Inserm U600, case 937, \\ 163 Avenue de Luminy 13288 Marseille Cedex 9, France \\ 2.CNRS UMR 6212 \\ 3.Aix Marseille University \\ 4.Laboratoire des Colloïdes Verres et Nanomatériaux UMR 5587, \\ CNRS/UM2, CC2 34095 Montpellier Cedex 5, France
}

(Dated: November 23, 2010)

\begin{abstract}
We show that the motion of individual red blood cells in an oscillating moderate shear flow is described by a nonlinear system of three coupled oscillators. Our experiments reveal that the cell tanktreads and tumbles either in a stable way with synchronized cell inclination, membrane rotation and hydrodynamic oscillations, or in an irregular way, very sensitively to initial conditions. By adapting our model described in ref [2], we determine the theoretical diagram for the red cell motion in a sinusoidal flow close to physiological shear stresses and flow variation frequencies and reveal large domains of chaotic motions. Finally, fitting our observations allows a characterization of cell viscosity and membrane elasticity.
\end{abstract}

PACS numbers: 47.52.+j; 83.50-v; 83.80.Lz; 87.85.G

Keywords: time dependent flow; tanktreading; swinging; tumbling; capsule; elasticity; Lyapunov exponent

Oscillators are ubiquitous in physics, chemistry (Belousov-Zhabotinski reaction) and in the living world at the scale of single organisms (pairs of beating eukaryotic flagella [1]) or of populations (fireflies blinking). Ever since Huygens noticed in 1665 that two pendulum clocks synchronized, scientists discovered that coupled oscillators showed a range of complex behaviors from synchronous to chaotic oscillations. Recently, we demonstrated that the movement of a red blood cell (RBC) in a steady shear flow is well described by two ordinary equations analog to the problem of two dissipative coupled angular oscillators represented by the cell inclination angle relatively to the flow direction and the rotation angle of the membrane elements around the center of mass of the cell [2]. The two angles are synchronized, as required for $2 \mathrm{D}$ non-linear systems [2-4]. In the vasculature, however, the blood flow is time-dependent. In arteries it is pulsed and in the microcirculation, it presents irregular temporal variations, from intermittent cessation in capillary flows to chaotic behavior in arterioles [5-7]. These fluctuations, which are partly related to fluctuations of vascular calibre are still poorly understood. At the RBC scale, the time-dependent external flow plays the role of a third coupled oscillator, which, by adding a degree of freedom to the non-linear system could allow the cell to have a complex, even chaotic motion. Such motion would in turn influence the large scale flow of blood and contribute to its observed temporal fluctuations. In this work, we provide a theoretical description of the motion of RBCs in a sinusoidal moderate shear flow close to physiological shear stresses and flow variation frequencies that we illustrate with several experiments. We predict that RBCs can present a chaotic motion and we experimentally show the unstable movement of RBCs. We propose a full theoretical diagram of the RBC motion as a function of the amplitude and the frequency of the shear rate. We show that the existence of a cell shape memory associated with a minimum of the elastic energy of the membrane for a specific position of its elements is crucial for chaos. Finally, as our experiments allow an easy recording of RBC motion they may provide a suitable tool to characterize cell viscosity and elasticity.

Model - The motion under a moderate shear flow of a $\mathrm{RBC}$ whose symmetry axis lies in the shear plane is characterized by two angles: $\theta$, the cell inclination angle with respect to the flow direction and $\omega$, the angle between the instantaneous position of a membrane element and its initial position at rest (Fig. 1). At low shear stress, RBCs tumble $(\mathrm{T})$, i. e. $\theta$ rotates and $\omega$ oscillates. Above critical values of the external viscosity and the shear stress, the cell inclination stabilizes ( $\theta$ oscillates around a mean value) while the membrane rotates around the fixed cell shape ( $\omega$ rotates): the cell both tanktread (TT) and swing $(\mathrm{S})$. The membrane elements, displaced during the tanktreading revolution are deformed and store an elastic energy which reaches a maximum for every $\pi$ rotation of the membrane. This energy barrier modifies periodically the energy balance of the system and is at the origin the $\theta$-oscillations (Fig. 1). At the T-STT transition, there is an intermittent regime with alternative series of T and STT $[2,8,9]$, where the numbers of T and STT depend on the shear rate. A recent analytical simple model derived from Keller and Skalak's (KS) [10] was proposed $[2,9]$, which treats a $\mathrm{RBC}$ as a viscoelastic ellipsoidal membrane enclosing a viscous fluid. It does not account for cell deformation and is therefore adapted to moderate shear stress. It deals with a basic mechanical description of the membrane and a simplified velocity field on the membrane, which lead to tractable analytical equations that retrieved the observed regimes of cell 
motion and provided semi-quantitative fits of experimental data [2]. Subsequent full numerical models [11-13] derived from this approach basically retrieved the same features for the cell motion. The RBC shape is described by a fixed oblate ellipsoid filled with a Newtonian solution of viscosity $\eta_{i}$ and delimited by a viscoelastic thin membrane, which obeys a simple Kelvin-Voigt constitutive law: $\sigma=2 \eta_{m} \mathbf{D}+2 \mu_{m} \mathbf{E}$, where $\eta_{m}$ and $\mu_{m}$ are the membrane viscosity and shear modulus and $\mathbf{D}$ and $\mathbf{E}$ are the strain rate and strain tensors, respectively. The sinusoidal shear rate of the flow is $\dot{\gamma}=\dot{\gamma}_{a} \sin (\nu t)$. The balance of torques exerted by the external fluid on the cell and the conservation of energy [10] - the rate of viscous dissipation in the cell plus the elastic power stored by the cytoskeleton is equal to the power provided by the external fluid on the cell - yield the two equations for RBC motion given in ref [2]. These equations still hold for time-dependent shear rate. Adding a third equation with $\tilde{\nu}=\nu t$ yields a $3 \mathrm{D}$ autonomous flow $F(\vec{X})$ such as $d \vec{X} / d t=F(\vec{X})$, where $\vec{X}$ is the vector $(\theta, \omega, \tilde{\nu})$ :

$$
\begin{aligned}
\partial_{t} \theta= & \dot{\gamma_{a}} \sin (\tilde{\nu})\left(-\frac{1}{2}+\frac{a_{1}^{2}-a_{2}^{2}}{a_{1}^{2}+a_{2}^{2}} \cos (2 \theta)\right)-\frac{2 a_{1} a_{2}}{a_{1}^{2}+a_{2}^{2}} \partial_{t} \omega \\
\partial_{t} \omega= & \frac{f_{3} \dot{\gamma_{a}}}{f_{2}-\frac{\eta_{i}}{\eta_{o}}\left(1+\frac{\eta_{m}}{\eta_{i}} \frac{\Omega}{V}\right) f_{1}} \\
& \quad \times\left[\frac{f_{1}}{2 f_{3}} \frac{\mu_{m}}{\eta_{o} \dot{\gamma_{a}}} \frac{\Omega}{V} \sin (2 \omega)-\sin (\tilde{\nu}) \cos (2 \theta)\right] \\
\partial_{t} \tilde{\nu}= & \nu
\end{aligned}
$$

$f_{1}, f_{2}$ and $f_{3}$ are geometrical constants described in ref [10], $\mathrm{a}_{1}$ and $\mathrm{a}_{2}$ are the lengths of the long and small axis of the cell cross-section, $\mathrm{V}$ is the $\mathrm{RBC}$ volume, $\Omega$ is the membrane volume and $\eta_{o}$ is the external suspending fluid viscosity. The phase space is conveniently described by the ensemble $(\theta, \omega, \sin (\tilde{\nu}))$. Equations are solved numerically by using the set of parameters [2]: $\mathrm{a}_{1}=\mathrm{a}_{3}=4 \mu \mathrm{m}$, $\mathrm{a}_{2}=1.5 \mu \mathrm{m}, \frac{\Omega}{V}=7.48 .10^{-2}, \eta_{0}=34.10^{-3}$ Pa.s, $\eta_{i}=$ $10^{-2}$ Pa.s, $\eta_{m}=0.7$ Pa.s, $\mu_{m}=1.6 \mathrm{~Pa}$.

For small shear rate $\left(\dot{\gamma}_{a}<\dot{\gamma}_{c}\right)$, where $\dot{\gamma}_{c}$ is the critical shear rate of the steady T-STT transition, RBCs tumbles periodically. The phase trajectories approach a stable limit-cycle as shown in Fig. 1a on the projection on the plane $(\theta, \omega)$. The approach to the limit-cycle takes about 1 sec. The angle $\theta$ changes monotonously with time: clockwise during the forward movement $(\dot{\gamma}>0)$ and counterclockwise back to its initial value during the backward movement $(\dot{\gamma}<0)$. The angle $\omega$ oscillates, indicating that the membrane slightly moves back and forth about its position at rest.

For larger values of the shear rate amplitude $\left(\dot{\gamma}_{a}>\dot{\gamma}_{c}\right)$, the cells swing and tanktread when the instantaneous shear rate is larger than $\dot{\gamma}_{c}$ and tumble when it decreases below $\dot{\gamma}_{c}$ just before the flow changes direction, as shown
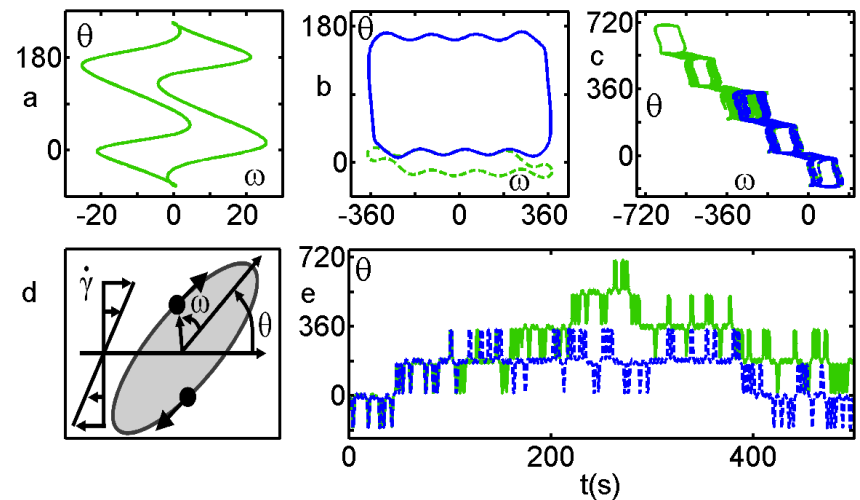

FIG. 1: a-c: numerical simulation of $\theta\left(^{\circ}\right)$ vs. $\omega\left({ }^{\circ}\right)$. a: limit cycle $\dot{\gamma}_{a}=2 s^{-1}, \nu=0.2 \mathrm{rd} . \mathrm{s}^{-1}$. b: two limit cycles $\dot{\gamma}_{a}=$ $9 s^{-1}, \nu=0.35$ rd.s $s^{-1}$; Initial conditions: $\omega_{0}=0, \theta_{0}=0(-)$ or $\theta_{0}=\pi / 2(--)$. c: non-closed trajectory for $\dot{\gamma_{a}}=9 \mathrm{~s}^{-1}$, $\nu=0.8 \mathrm{rd.s} \mathrm{s}^{-1}$, initial conditions: $\omega_{0}=0, \theta_{0}=10^{-9}(-)$ or $\theta_{0}=-10^{-9}(--)$. d: sketch of a RBC in the flow. e: numerical chaotic trajectories $\theta\left({ }^{\circ}\right)$ vs. time, initial conditions of $c$.

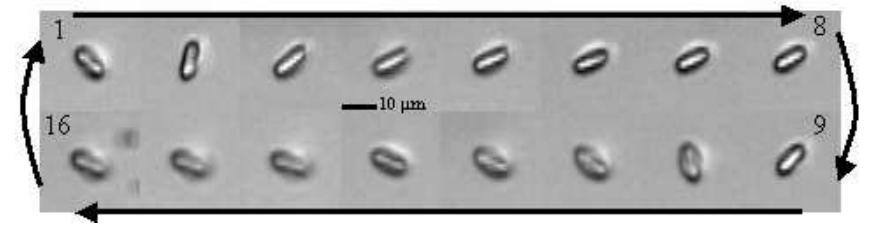

FIG. 2: Sequence of a RBC round trip during one period of the flow. Forward motion 1- 8, snapshot times (in s): 0, $0.32,0.64,1.08,2.12,3.28,3.72,4.16$; backward motion 9- 16 , snapshot times: $4.56,4.88,5.16,5.76,7.08,7.98,8.24$ and 8.8

on an experimental sequence in Fig. 2. The model predicts a stable periodic regime described by either one or two non-intersecting limit-cycles (see Fig. 1b), whose trajectories are determined by the initial angles. During the S-TT regime, $\theta$ is little sensitive to the initial conditions. However, the storage of elastic energy slightly differs on each trajectory. Therefore the TT-T transition does not occur at the same moment and the values of $\theta$ and $\omega$ when the flow changes direction differ from one trajectory to the other. The consequence for the cell reversal is major: in one case, at the end of the forward movement the cell has a counterclockwise reversal and passes vertically (see Fig. 2), perpendicularly to the flow direction, whereas in the other case, the cell rotates in the clockwise direction and passes horizontally, in the alignment of the flow direction.

For specific values of $\dot{\gamma}_{a}$ and $\nu$, a non-periodic motion, highly sensitive to initial conditions and characterized by an unstable limit-cycle occurs. Projections of portions of trajectories are shown in Fig. 1c. Trajectories are no longer closed and two initially very close initial conditions lead to strongly divergent trajectories. For illustrations of the attractor and first return map see [14]. In this case, the three oscillators $\theta, \omega$ and $\dot{\gamma}$ cannot synchronize and 
the movement is chaotic. Chaos was further investigated by computing the Lyapunov exponents of the system, which characterize the rate of separation of initially close trajectories. Let $\vec{X}(t)$ be a solution of the flow $\mathrm{F}$ and $\delta \vec{X}(0)$ an initial infinitesimal separation. The separation at time t, $\delta \vec{X}(t)$, obeys the equation of evolution obtained by linearisation of the flow $\mathrm{F}$, and its integration leads to the exponential evolution of the perturbation $\delta \vec{X}(t)$ :

$$
\frac{d \delta \vec{X}}{d t}=\left.\frac{\partial F}{\partial \vec{X}}\right|_{X \overrightarrow{(t)}} \delta \vec{X} ; \quad \delta \vec{X}(t)=\delta \vec{X}(0) e^{\left.\int_{0}^{t} \frac{\partial F}{\partial \vec{X}}\right|_{X \overrightarrow{(t)}} d t}
$$

For each given solution, we computed the Jacobian matrix $\partial F / \partial \vec{X}$, we numerically determined the matrix $\left.\int_{0}^{t} \frac{\partial F}{\partial \vec{X}}\right|_{X(t)} d t$, and we calculated its three eigenvalues at several times. The slopes of the asymptotic linear variations of these values with time are the three Lyapunov exponents of the system. The one corresponding to the parameter $\tilde{\nu}$ is equal to 0 and the second one is always negative. The largest exponent $\lambda$ may be positive (see [14]), which is a signature of chaotic motion. Variations of $\lambda$ and of the error on $\lambda$ (determined from the correlation coefficient of the least square fit, $1-r^{2}$ ) with the shear rate frequency are shown in the insert of Fig. 3 . The error is small for the largest positive values of $\lambda$, thus confirming the existence of chaotic zones of the RBC motion. We analyzed the Floquet matrix for the Poincare section computed when the instantaneous shear rate equals 0 at the bifurcation event. The Floquet eigenvalue crosses the unit circle along the real axis at +1 , wich characterizes a saddle-node bifurcation and discloses that the switch to chaotic behavior occurs via an intermittency (type I) mechanism, characterized by bursts of chaos. We fully characterized the RBC dynamical behavior by plotting $\lambda$ as a function of $\nu$ and the RMS value of the shear rate $\dot{\gamma}_{r m s}=\dot{\gamma}_{a} / \sqrt{2}$ (Fig. 3). Values for $\nu$ span physiological frequencies, from vasomotion fluctuations (tenths of Hertz) to the heart frequency (one Hertz) and values of $\dot{\gamma}$ cover the range of stresses $\eta_{o} \dot{\gamma}$ observed in the vaculature, from capillaries to arteries [5-7]. When $\dot{\gamma}_{r m s}$ decreases below $3.1, \lambda$ deeply falls around -0.5 , revealing that the system is stable. The value 3.1 is equal to $\dot{\gamma}_{c}$, the critical shear rate of the T-TT transition in constant shear flow for the RBC we computed. Tumbling stabilizes the motion. Indeed, during tumbling, the cell membrane is 'quasi-solidified', the angle $\omega$ remains very small and it can be eliminated in the first equation of eq. 1 by using the second equation where the term in $\sin (2 \omega)$ is neglected. The system then loses one dimension and chaos is no longer possible. Above $\dot{\gamma}_{c}$ the motion is much less stable. Unstable chaotic zones appear. They form widening bands located on a bundle of lines. The solid line represents the $\left(\dot{\gamma}_{r m s}, \nu\right)$ couples for which a RBC performs a full swinging cycle during the tanktreading time of the forward motion $(\dot{\gamma}>0)$ and one swinging cycle during the backward motion $(\dot{\gamma}<0)$. The other dotted
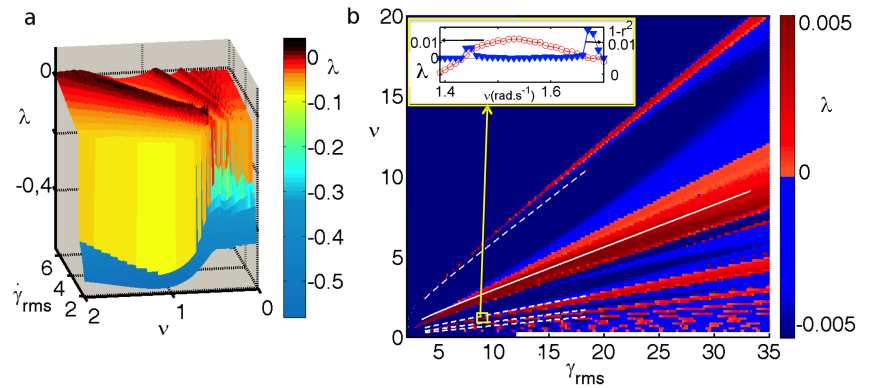

FIG. 3: Diagram of the dynamical RBC motion; a: 3D map of $\lambda$ vs. $\nu$ and $\dot{\gamma}_{r m s}$; b: 2D projection: values of $\lambda$ (in color) in the $\left(\nu, \dot{\gamma}_{r m s}\right)$ plane. Dark regions (positive $\lambda$ ) are chaotic zones. Solid line: flow conditions for which RBCs perform a full swinging cycle during the forward (or backward) motion. Dotted lines from top to bottom: RBCs perform respectively $1 / 2,2,3$ and 4 swinging cycles during the forward motion; insert: variation of $\lambda$ and $1-r^{2}$ with $\nu$ in a chaotic zone.

lines characterize the flow conditions for which a RBC swings respectively $1 / 2$ (steepest line), 2, 3 and 4 times during half a flow period. The strong correlation between these lines and the chaotic bands shows that chaos appears at the resonance of the flow frequency with the internal specific cell frequency. As expected, chaos is intimately related to swinging, i. e. to the elastic storage of the non spherical membrane, since the term with the elastic modulus $\mu_{m}$ of Eq.1 induces the coupling of the three differential equations. Viscous capsules or spherical viscoelastic capsules must present a stable dynamics in a time-dependent shear flow.

Observations - Direct measurements of $\theta$ are provided from side-view microscopic imaging in a vertical plane parallel to the shear plane $[2,15]$. Sinusoidal flow was created from the variation of hydrostatic pressure by connecting the flow chamber to a reservoir $(10 \mathrm{ml})$ bound to the outer edge of a vertical rotating wheel (radius $9.45 \mathrm{~cm})$. The frequency of rotation of the wheel $\nu$ (in $\mathrm{rd} / \mathrm{s}$ ) was varied in the range $[0.16-1.15 \mathrm{rd} / \mathrm{s}]$ and $\dot{\gamma}_{a}=6.72 \mathrm{~s}^{-1}$. RBCs were diluted in a solution of dextran (concentration $9 \% \mathrm{w} / \mathrm{w}, \eta_{o}=34 \mathrm{mPa} . \mathrm{s}, \mathrm{Mw}=2.10^{6}$ $\mathrm{g} / \mathrm{Mol}$ ) with $\mathrm{PBS}$ at $290 \mathrm{mOsm}$ and $\mathrm{pH}=7.4$. RBCs were almost non-buoyant in the solution and could flow during several hours without sinking. The membrane shear modulus as well as the internal and membrane viscosities of RBCs depend on the age of the cell and are therefore widely distributed when measured in a blood sample. The maximal applied shear stress $\eta_{o} \dot{\gamma}_{a}$ was specifically chosen to be larger than the critical shear stress $\eta_{o} \dot{\gamma}_{c}$ for the majority of RBCs, so that most cells swing and tanktread but the stiffest ones tumble. We recorded cell movements 10 minutes after application of the flow in order to let the motion stabilize and reach the limit-cycle. We observed the three motions predicted by the model. Stable tumbling is characterized by the continuous variation of $\theta$ with time as illustrated in Fig. 4a over three flow 


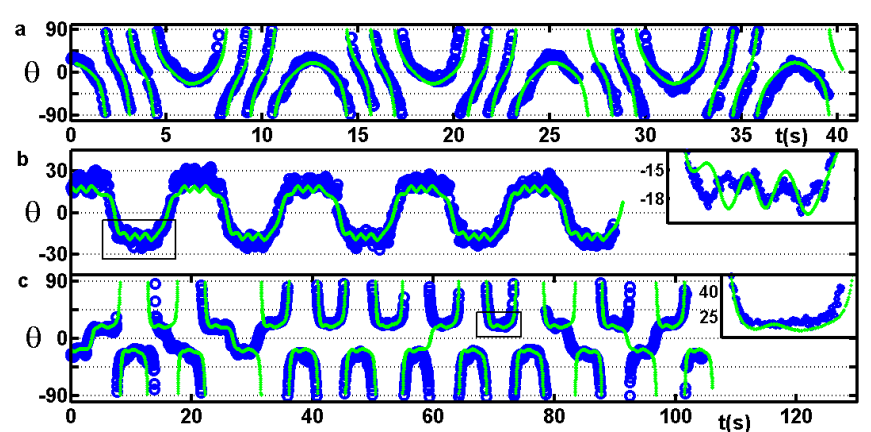

FIG. 4: Experimental variation and fits of $\theta$ vs. time for 3 RBCs. a: $\nu=0.08 r d . s^{-1}$, stable T, fit $\eta_{\text {eff }}=1.2510^{-2}$ Pa.s, $\mu=15.2 P a ; \mathrm{b}: \nu=0.05 r d . s^{-1}$, stable S-TT and T, fit $\eta_{e f f}=$ $4.9510^{-2}$ Pa.s, $\mu=0.591 P a$; c: $\nu=0.11 \mathrm{rd}_{\mathrm{d}} \mathrm{s}^{-1}$. Unstable chaotic motion, fit $\eta_{\text {eff }}=3.9710^{-2}$ Pa.s, $\mu=0.49 P a$. Inserts show a zoom of the boxed regions

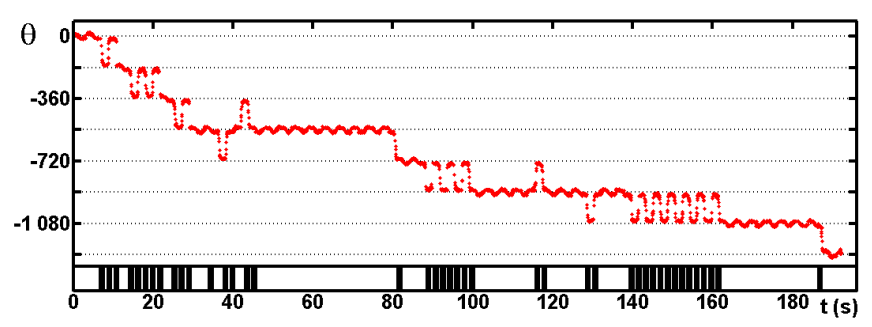

FIG. 5: long experiment: variations of the absolute value $\theta$ vs. time. Bottom: the black lines denotes vertical reversals

periods. For $\dot{\gamma}_{a}>\dot{\gamma}_{c}$, the stable regime is shown on two cells, in Fig. 4b and in Fig. 2, which displays snapshots of a typical sequence, where one can notice that the cell shape does not significantly change during the motion. The cell swings and tanktreads at high shear rate and tumbles when the shear rate decreases below $\dot{\gamma}_{c}$, when the flow changes direction. The modulation of the shear rate allows the $\mathrm{RBC}$ to exhibit alternatively a solid-state and a viscoelastic capsule-like dynamical behavior during the time flow. Unstable non-periodic regime was also observed as shown in Fig. 4c and in Fig. 5 over more than 50 periods. As we predicted theoretically: the way the cell rotates to change direction varies with time. The cell non-periodically alternates sequences of horizontal reversals $(\theta=0)$ and vertical reversals $(\theta=\pi / 2)$. The consequence is that the number of clockwise and anticlockwise rotations are different as illustrated in Fig. 5 where the cell finally presents a net clockwise tumbling motion although the mean flow is equal to zero.

Finally, our model and our experiments are in good semi-quantitative agreement: experimental curves $\theta(t)$ are well-fitted by our model by adjusting the two parameters, $\mu_{m}$ and $\eta_{e f f}=\eta_{i}+\eta_{m} \frac{\Omega}{V}$ as shown in Fig. 4 and in [14] for a single cell observed at 6 different flow frequencies. In the case of unstable behavior, an adjustement is stricto sensu not possible since the motion is highly sensitive to initial conditions but the theoretical curve well describes the main characteristics of the motion (see inserts of Fig.4) and predicts the instability of motion.

Here, we predict that dilute RBCs present a chaotic motion over large domains of external sinusoidal flows, under shear stresses and flow variation frequencies relevant for arterial and microcirculation physiology. In-vivo, in the whole blood cells form a concentrated suspension and interact together. These interactions couple more oscillators together and should result in enhanced chaotic motions which could affect flow properties in the sheared blood layer close to vessel walls. Finally, our approach also applies to other physical systems used in flow as polymer capsules or protein-coated drops.

We thank Dr. R. Bruinsma for his suggestions, P. Soulé for discussions and C. Meuzard for experiments. Our labs belong the CNRS French Consortium 'CellTiss'.

* Electronic address: annie.viallat@inserm.fr

[1] R. E. Goldstein, M. Polin, and I. Tuval, Phys. Rev. Lett., 103, 168103 (2009)

[2] M. Abkarian, M. Faivre, and A. Viallat, Phys. Rev. Lett., 98, 188302 (2007)

[3] H. Noguchi, Phys. Rev. E, 80, 021902 (2009)

[4] S. Kessler, R. Finken and U Seifert, Eur.. Phys. J. E, 29, 399 (2009)

[5] T. M. Griffith, Cardiovascular Research, 31, 342 (1996) doi:10.1016/S0008-6363(95)00147-6

[6] J. Trzeciakowski and W. M. Chilian, Med. Biol. Eng. Comput., 46, 433 (2008)

[7] K. D. Barclay, G. A. Klassen and C. Young, Microcirculation, 7, 335 (2000)

[8] M. Abkarian and A. Viallat, Soft Matter, 4, 653 (2008)

[9] J. M. Skotheim and T. W. Secomb, Phys. Rev. Lett., 98, 078301 (2007).

[10] S. Keller and R. Skalak, J. Fluid Mech. 120, 27 (1982).

[11] S. Kessler, R. Finken and U. Seifert. J. of Fluid Mech., 605, 207 (2008)

[12] H. T. Low, Y. Sui, Y. T. Chew and P. Roy, Modern Physics Letters B, 23, No. 3, 545 (2009)

[13] Y. Sui, Y. T. Chew, P. Roy, Y. P. Cheng and H. T. Low,, Physics of Fluids, 20, 112106 (2008)

[14] supplementary information

[15] M. Abkarian and A. Viallat, Biophys. J. 89, 1055 (2005).

[16] H. Schmid-Schonbein and R. Wells, Science, 165, 288 (1969)

[17] H. Goldsmith, and J. Marlow, Proc. R. Soc. Lond. B. 182, 351 (1972)

[18] T. Fischer and H. Schmid-Schönbein, Blood Cells 3, 351 (1977).

[19] T. Fischer, M. Stöhr-Liesen, and H. Schmid-Schönbein, Science 202, 894 (1978).

[20] R. Tran-Son-Tay, S. Sutera, and P. Rao, Biophys. J. 46, 65 (1984).

[21] P. Bagchi and R. M. Kalluri, Phys. Rev. E, 80, 016307 (2009)

[22] N. Watanabe, H. Kataoka, T. Yasuda, and S. Takatani, Biophys. J. , 91, 1984 (2006)

[23] N. Mohandas and E. Evans, Annu. Rev. Biophys. Biomol. 
Struct., 23, 787 (1994). 